\title{
On-Line Appendix for: The Cyclical Volatility of Labor Markets under Frictional Financial Markets
}

\author{
By Nicolas Petrosky-Nadeau and Etienne Wasmer*
}

\section{NumericAl APPENDIX}

The basic unit of time is a month. The matching function in the labor market is assumed to take the usual form $M_{l}(\mathcal{V}, u)=\chi \mathcal{V}^{1-\eta} u^{\eta}$, where $\chi>0$ is a level parameter. We set $\eta=0.6$, drawing on the survey of estimates of the labor matching function in Petrongolo and Pissarides (2001) and advocated by Brügemann (2008). We set the job separation rate, $s$, to 0.03 , corresponding to the monthly value reported for the U.S. in Davis et al. (2006) based on the Jobs Openings and Labor Turnover Survey (JOLTS). We target an unemployment rate of 7\%, as in Gertler and Trigari (2009), which implies a job finding rate $f=s(1-u) / u$. We target a job filling rate $q=0.4$ based on the estimates for the U.S. in Den Haan et al. (2000). The constant returns of the matching function implies that labor market tightness is $\theta=f / q$, and steady state job vacancies can be calculated as $\mathcal{V}=\theta u$ given our target for the unemployment rate.

Silva and Toledo (2007) estimate costs related to recruiting a worker of $3.6 \%$ of the wage rate, $\operatorname{or} \gamma \mathcal{V} / w=0.036$. In combination with a target for a labor share of national income, $w / y$, of two thirds (Golin, 2002), the unit vacancy costs is calculated as $\gamma=0.036 w / \mathcal{V}$.

The economy's job creation condition then determines the value of $\Gamma$ as $\Gamma=$ $q(y-w) /(r+s)$, where the variable $\Gamma$ is defined as $\Gamma=\gamma+\frac{r+q}{1+r} K$. Consequently, we can compute $K=(\Gamma-\gamma)(1+r) /(r+q)$. Recall the wage equation $w=$ $\alpha[y+\Gamma \theta]+(1-\alpha) z$. Assuming $z=0.4$, as in Shimer (2005), the bargaining weight $\alpha$ that satisfies our targeted wage is

$$
\alpha=\frac{w-z}{y+\Gamma \theta-z} .
$$

The specification of the credit market requires choosing five parameters: the costs of prospecting on credit markets, $\kappa$ and $e$, the bargaining weight $\beta$ and the parameters of the credit matching function, $M_{c}(\mathcal{B}, \mathcal{E})=\varsigma^{1-\epsilon} \mathcal{B}^{\epsilon}$, a level $\varsigma>0$

* Petrosky-Nadeau: Tepper School of Business, Carnegie Mellon University, 5000 Forbes Ave., Pittsburgh, PA, 15213. E-mail: npn@cmu.edu. Wasmer: Sciences Po, Paris, Département d'Economie, 28 Rue des Saint-Pères, 75007 Paris, France. E-mail: etienne.wasmer@sciences-po.fr. 
and elasticity $0<\varepsilon<1$. For lack of direct evidence, we set the elasticity of the credit market matching function to $\epsilon=0.5$, and assume a symmetry in the cost of prospecting the credit market, i.e., $\kappa=e$. Our strategy is then to use an observed size of the financial sector to determine the weight $\beta$, constrain the level parameter $\zeta$ by limiting the duration of search in the credit market and the search $\operatorname{cost} \kappa$ is determined by the previously calculated costs $K$.

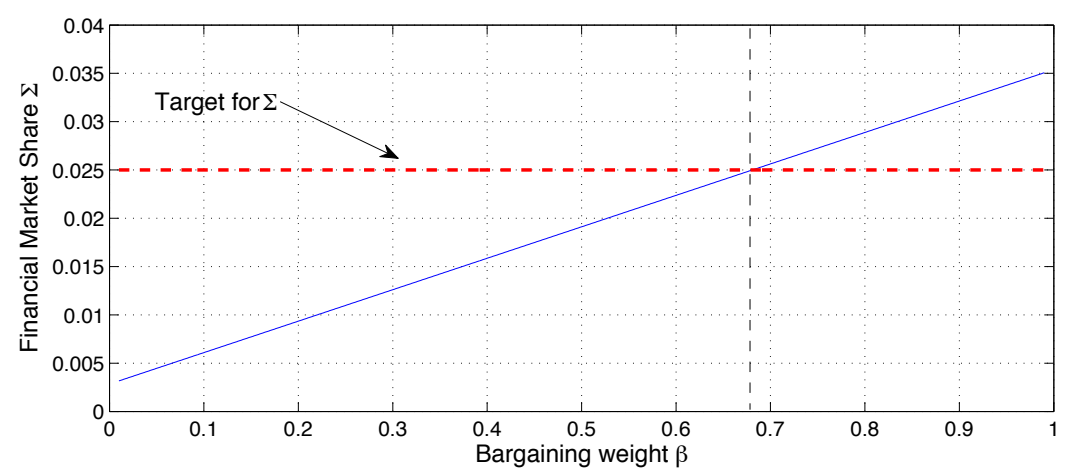

Figure A1. Financial Sector's Share of GDP as a Function of $\beta$

Our main target for the financial market is its share of aggregate value added:

$$
\Sigma=\frac{(1-u) \Psi-\gamma \mathcal{V}-\mathcal{B} \kappa}{1-u}
$$

where $\Psi=\beta[y-w]+(1-\beta) \frac{\gamma}{q}(r+s)$ and $\mathcal{B}=\frac{q \mathcal{V}}{\phi p(\phi)}$. Substituting $\mathcal{B}$ we have

$$
\Sigma=\frac{(1-u) \Psi-\left(\gamma+\frac{q \kappa}{\phi p(\phi)}\right) \mathcal{V}}{1-u}
$$

Recall that $K=\frac{e}{p(\phi)}+\frac{\kappa}{\phi p(\phi)}$ and notice that

$$
K=\frac{\phi e+\kappa}{\phi p(\phi)}=\frac{\frac{1-\beta}{\beta} \kappa+\kappa}{\phi p(\phi)}=\frac{\frac{1}{\beta} \kappa}{\phi p(\phi)} \Rightarrow \frac{\kappa}{\phi p(\phi)}=\beta K
$$

which we can substitute into the definition for $\Sigma$ :

$$
\Sigma(\beta)=\frac{(1-u)\left[\beta[y-w]+(1-\beta) \frac{\gamma}{q}(r+s)\right]-(\gamma+q \beta K) v}{1-u}
$$


Given our targets and parameter values so far, $\Sigma$ is an increasing function of $\beta$ and we can pin down $\beta$ with our target for $\Sigma=0.025$, as illustrated in Figure 1.

The functional form for the matching function in the credit market implies meeting rates $p(\phi)=\zeta \phi^{-\epsilon}$ and $\phi p(\phi)=\zeta \phi^{1-\epsilon}$. Assuming symmetry in search costs, $\kappa=e$, credit market tightness $\phi=\frac{1-\beta}{\beta} \frac{\kappa}{e}$ can now be computed as $\phi=$ $(1-\beta) / \beta$. We target an average duration in the credit market of 4 months $1 / \phi p(\phi)=4$. Then the level parameter in the credit market matching function is $\zeta=1 /\left[4 \phi^{1-\epsilon}\right]$. The we can obtain $p(\phi)=\zeta \phi^{-\epsilon}, \kappa=\beta K \phi p(\phi)$ and $e=$ $(1-\beta) K p(\phi)$. This completes the parameterization of the model.

\section{A1. Additional Tables}




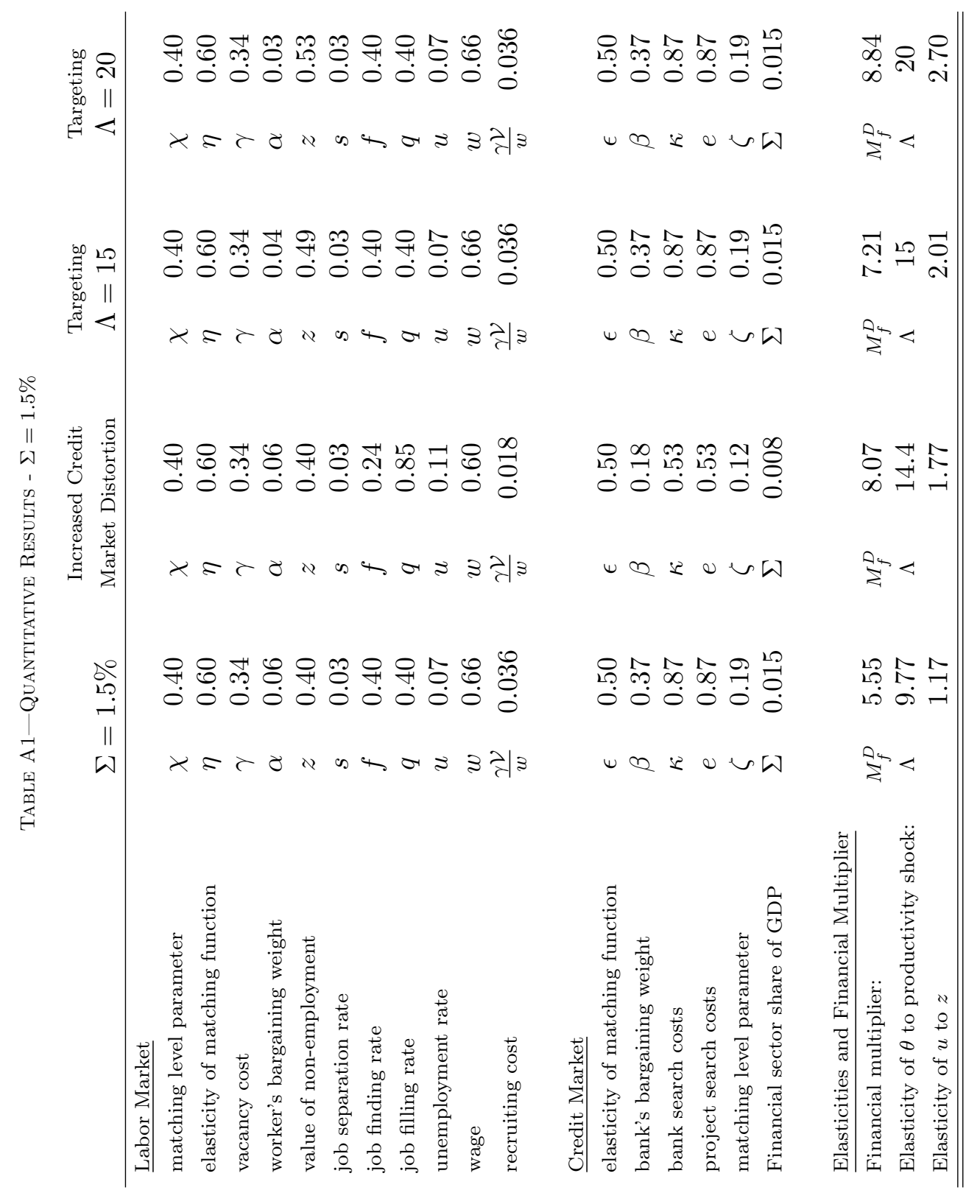




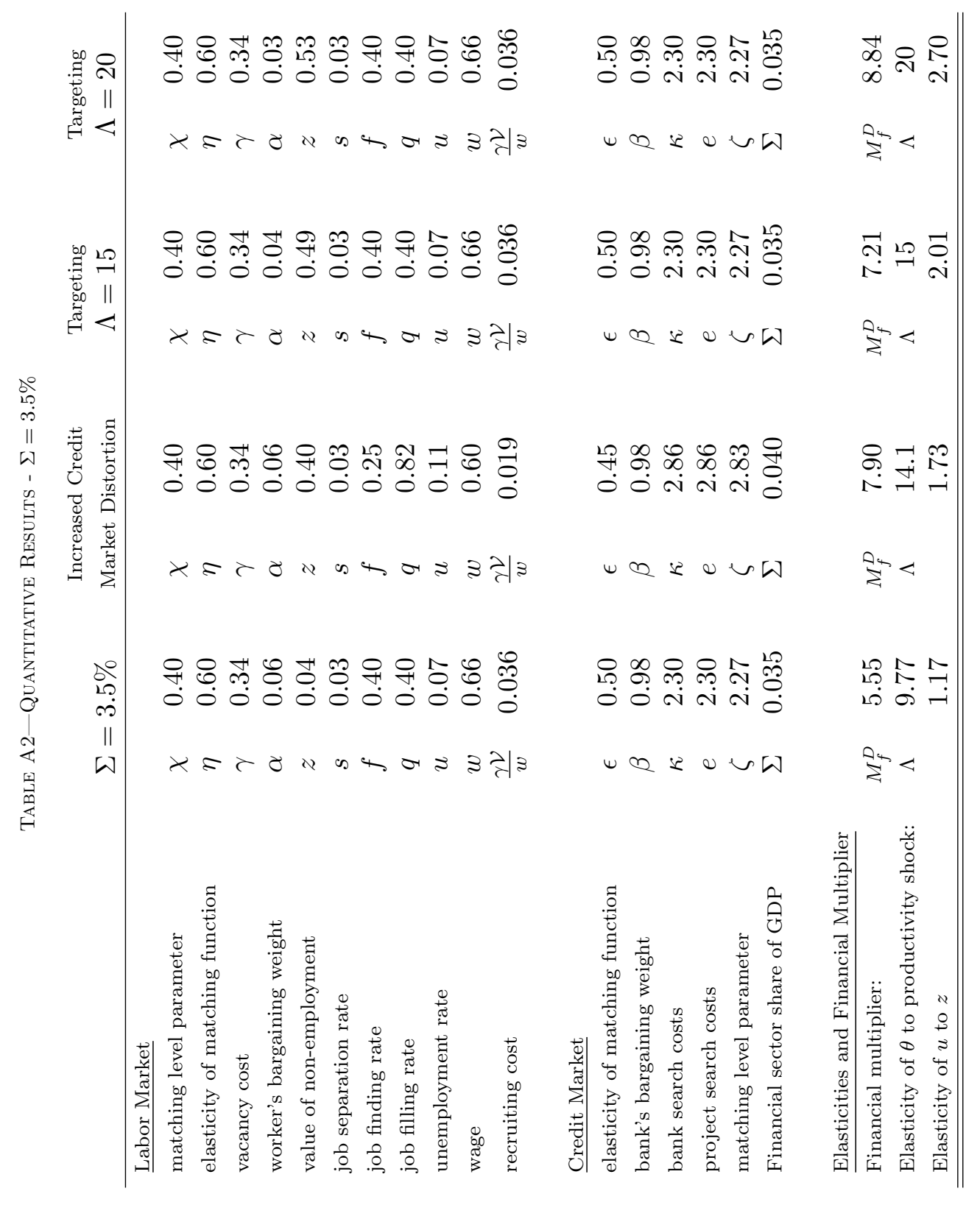


Table A3-Quantitative Results - Different Job Filling Rates $q$

\begin{tabular}{|c|c|c|c|c|c|c|}
\hline \multirow{2}{*}{ Labor Market } & \multicolumn{2}{|c|}{$\begin{array}{c}\text { Baseline } \\
q=0.4\end{array}$} & \multicolumn{2}{|c|}{$\begin{array}{c}\text { Lower } \\
q=0.2\end{array}$} & \multicolumn{2}{|c|}{$\begin{array}{l}\text { Higher } \\
q=0.7\end{array}$} \\
\hline & & & & & & \\
\hline matching level parameter & $\chi$ & 0.40 & $\chi$ & 0.30 & $\chi$ & 0.50 \\
\hline elasticity of matching function & $\eta$ & 0.60 & $\eta$ & 0.60 & $\eta$ & 0.60 \\
\hline vacancy cost & $\gamma$ & 0.34 & $\gamma$ & 0.17 & $\gamma$ & 0.60 \\
\hline worker's bargaining weight & $\alpha$ & 0.06 & $\alpha$ & 0.06 & $\alpha$ & 0.06 \\
\hline value of non-employment & $z$ & 0.40 & $z$ & 0.4 & $z$ & 0.40 \\
\hline job separation rate & $s$ & 0.03 & $s$ & 0.03 & $s$ & 0.03 \\
\hline job finding rate & $f$ & 0.40 & $f$ & 0.40 & $f$ & 0.40 \\
\hline job filling rate & $q$ & 0.40 & $q$ & 0.20 & $q$ & 0.70 \\
\hline unemployment rate & $u$ & 0.07 & $u$ & 0.07 & $u$ & 0.07 \\
\hline wage & $w$ & 0.66 & $w$ & 0.66 & $w$ & 0.66 \\
\hline recruiting cost & $\frac{\gamma \mathcal{V}}{w}$ & 0.036 & $\frac{\gamma \mathcal{V}}{w}$ & 0.036 & $\frac{\gamma \mathcal{V}}{w}$ & 0.036 \\
\hline \multicolumn{7}{|l|}{ Credit Market } \\
\hline elasticity of matching function & $\epsilon$ & 0.50 & $\epsilon$ & 0.5 & $\epsilon$ & 0.5 \\
\hline bank's bargaining weight & $\beta$ & 0.68 & $\beta$ & 0.64 & $\beta$ & 0.70 \\
\hline bank search costs & $\kappa$ & 1.58 & $\kappa$ & 1.47 & $\kappa$ & 1.64 \\
\hline project search costs & $e$ & 1.58 & $e$ & 1.47 & $e$ & 1.64 \\
\hline matching level parameter & $\zeta$ & 0.37 & $\zeta$ & 0.33 & $\zeta$ & 0.38 \\
\hline Financial sector share of GDP & $\Sigma$ & 0.025 & $\Sigma$ & 0.025 & $\Sigma$ & 0.025 \\
\hline \multicolumn{7}{|l|}{ Elasticities and Financial Multiplier } \\
\hline Financial multiplier: & $M_{f}^{D}$ & 5.55 & $M_{f}^{D}$ & 5.58 & $M_{f}^{D}$ & 5.54 \\
\hline Elasticity of $\theta$ to productivity shock: & $\Lambda$ & 9.77 & $\Lambda$ & 9.83 & $\Lambda$ & 9.75 \\
\hline Elasticity of $u$ to $z$ & & 1.17 & & 1.15 & & 1.18 \\
\hline
\end{tabular}

